\title{
Inorganic pyrophosphatase-nanodiamond conjugates hydrolyze pyrophosphate in human synovial fluid
}

\author{
Anastasiya V. Valueva1), Roman S. Romanov2), Sofia S. Mariasina²), Maxim S. Eliseev), Elena \\ V. Rodina $)^{*}$ \\ 1) Pharmaceutical Department, Pavlov Ryazan State Medical University, 9 Vysokovol'tnaya St., Ryazan, \\ 390026, Russia \\ 2) Department of Fundamental Medicine, Lomonosov Moscow State University, 31, build. 5, Lomonosovsky \\ prospect, Moscow, 119991, Russia \\ 3) V.A. Nasonova Research Institute of Rheumatology, Moscow, Kaschirskoe h. 34A, 115522, Russia \\ 4) Chemistry Department, Lomonosov Moscow State University, 1, build. 3, Leninskie Gory, Moscow, 119992 , \\ Russia
}

\section{Supporting Information}

Table S1. Parameters of phosphate release from the synthesized calcium pyrophosphate by PPase samples. Conditions: $50 \mathrm{mM}$ Tris-HCl, pH 7.5, $10 \mathrm{mg} \mathrm{ml}^{-1} \mathrm{Ca}_{2} \mathrm{P}_{2} \mathrm{O}_{7} \cdot 2 \mathrm{H}_{2} \mathrm{O}, 2 \mathrm{mM} \mathrm{MgCl}, 2 \mathrm{mM} \mathrm{CaCl}_{2}$, $0.01 \mu \mathrm{g} \mathrm{ml}^{-1}$ PPase.

\begin{tabular}{llcc}
\hline \multicolumn{1}{c}{ PPase samples } & {$\left[\begin{array}{c}{\left[\mathrm{P}_{\mathrm{i}}\right]_{\max }} \\
{[\mu \mathrm{M}]}\end{array}\right.$} & $\begin{array}{c}k \\
{\left[\mathrm{hr}^{-1}\right]}\end{array}$ \\
\hline Control & & $43 \pm 2$ & $0.01 \pm 0.01$ \\
Ec-PPase & Soluble & $48 \pm 2$ & $0.02 \pm 0.01$ \\
& Immobilized on ND-NH & $26 \pm 2$ & $0.02 \pm 0.01$ \\
& Immobilized on ND-NH- $\left(\mathrm{CH}_{2}\right)_{6}-\mathrm{NH}_{2}$ & $40 \pm 2$ & $0.03 \pm 0.01$ \\
Mt-PPase & $28 \pm 1$ & $0.13 \pm 0.02$ \\
& Soluble & $29 \pm 1$ & $0.06 \pm 0.01$ \\
& Immobilized on ND-NH & $32 \pm 1$ & $0.04 \pm 0.01$ \\
\hline
\end{tabular}




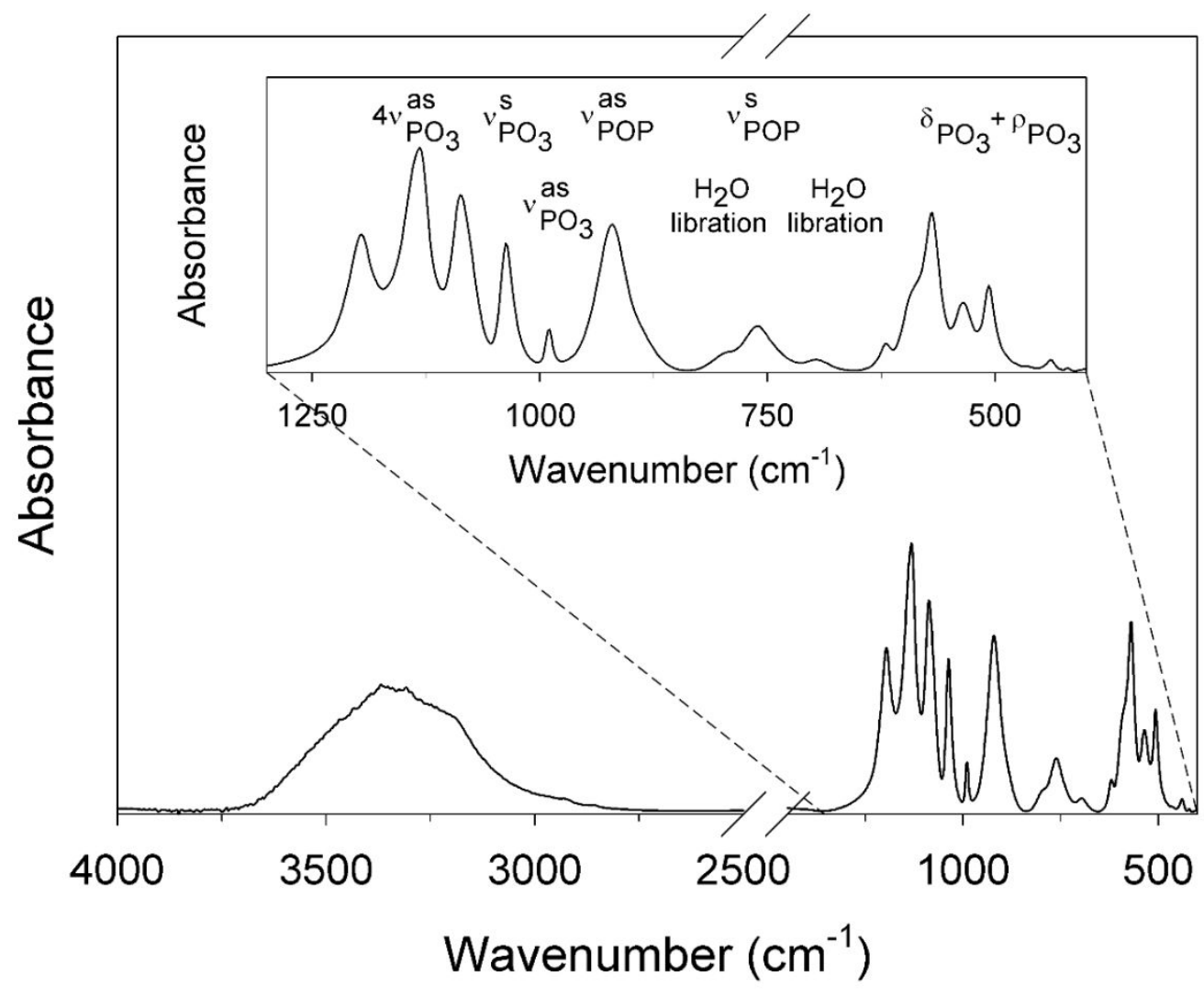

(a)

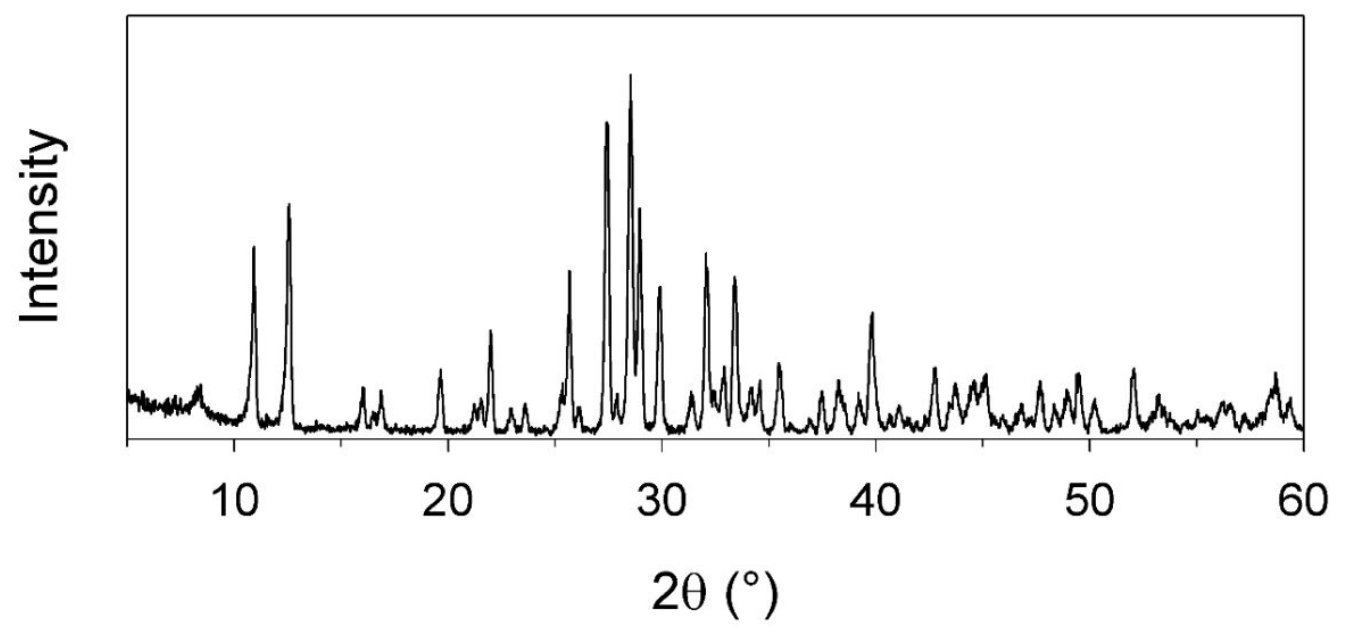

(b)

Figure S1. FTIR spectra of synthesized t-CPPD in the $4000-400 \mathrm{~cm}^{-1}$ region (a) and in the $1300-500 \mathrm{~cm}^{-1}$ region (inset). (b) XRD spectra of synthesized t-CPPD. 

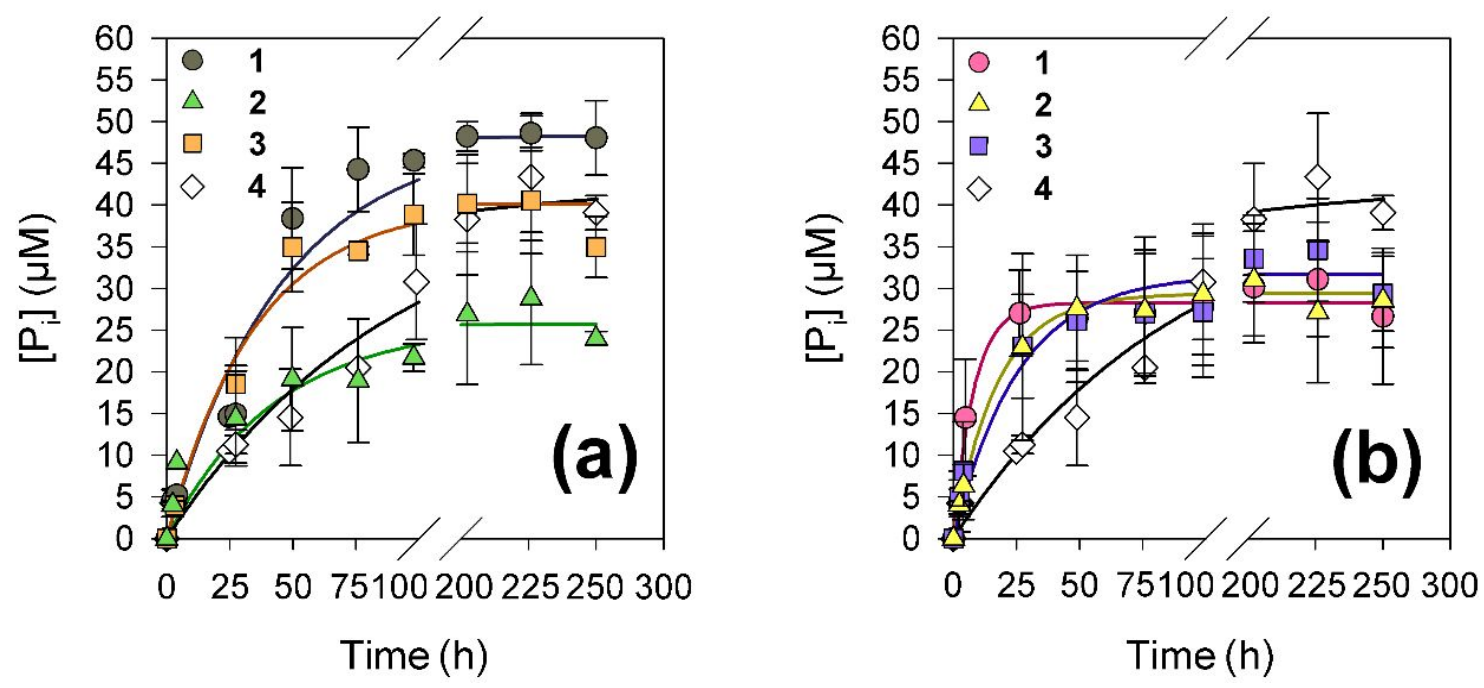

Figure S2. Hydrolysis of the synthesized calcium pyrophosphate by Ec-PPase (a) or Mt-PPase (b) in the soluble form (1) or immobilized on ND-NH$H_{2}$ (2) or ND-NH- $\left(\mathrm{CH}_{2}\right)_{6}-\mathrm{NH}_{2}$ (3). Conditions: $50 \mathrm{mM}$ Tris-HCl, pH 7.5, $10 \mathrm{mg} \mathrm{ml}^{-1} \mathrm{Ca}_{2} \mathrm{P}_{2} \mathrm{O}_{7} \cdot 2 \mathrm{H}_{2} \mathrm{O}, 2 \mathrm{mM} \mathrm{MgCl} 2,2 \mathrm{mM} \mathrm{CaCl}_{2}, 0.01 \mu \mathrm{g} \mathrm{ml}^{-1}$ PPase. Control experiment (4): the reaction mixture contained all these components except PPase. Lines are the best fit to the firstorder kinetics. 